$01,05,13$

\title{
Влияние внешних факторов на ширину линии ферромагнитного резонанса в структурах с обменным смещением
}

\author{
(С) И.О. Джунь, Г.В. Бабайцев, М.Г. Козин, И.Л. Ромашкина, Е.И. Шанова, Н.Г. Чеченин \\ Научно-исследовательский институт ядерной физики им. Д.В. Скобельцына \\ Московского государственного университета им. М.В. Ломоносова \\ Москва, Россия \\ E-mail: irina.dzhun@gmail.com
}

Поступила в Редакцию 5 сентября 2020 г.

В окончательной редакции 24 февраля 2021 г.

Принята к публикации 28 февраля 2021 г.

Исследованы внешние факторы, влияющие на ширину линии ферромагнитного резонанса (ФМР) в двухслойных (ферромагнетик/антиферромагнетик) системах с обменным смещением. Исследованы зависимость ширины линии ФМР от толщины антиферромагнитного (АФ) слоя при неизменной толщине ферромагнитного $(\Phi)$ слоя для образцов с различным порядком осаждения $\Phi$ - и АФ-слоев, а также корреляция между полем обменного смещения и шероховатостью поверхности образца. Обнаружено, что обменное смещение дает незначительный вклад в ширину линии ФМР. В системах с антиферромагнетиком, нанесенным на ферромагнитный слой, ширина линии ФМР увеличивается пропорционально среднему размеру шероховатости поверхности. В системах с обратным расположением слоев значительный вклад в ширину линии дает одноосная анизотропия. Ширина линии ФМР находится в квадратичной зависимости от одноосной анизотропии и в обратно пропорциональной зависимости от толщины антиферромагнитного слоя, что можно отнести к изменению микроструктуры с толщиной в качестве внешнего фактора демпфирования ФМР.

Ключевые слова: ферромагнитный резонанс, ширина линии, обменное смещение, одноосная анизотропия, шероховатость поверхности.

DOI: 10.21883/FTT.2021.06.50924.185

\section{1. Введение}

Исследование динамики намагниченности в наноразмерных магнитных структурах занимает важное место в сегодняшних исследованиях по нескольким причинам. В низкоразмерных устройствах, таких как сенсоры и спиновые диоды, принцип действия которых основан на эффекте гигантского магнитного сопротивления (ГМС), скорость переориентации намагниченности слоя является ключевым фактором. Динамика оценивается затуханием прецессии намагниченности относительно ее равновесного состояния [1-3]. Значение спинового затухания определяет ширину линии ФМР, которая является важной характеристикой для разработки устройств. В свою очередь, величина затухания зависит от ряда факторов, которые можно условно разделить на внешние и внутренние $[4,5]$. Среди внутренних факторов наиболее значительным является спин-орбитальное взаимодействие, которое определяется конфигурацией и размером магнитокристаллического поля (гильбертовское затухание) [6].

При использовании различных методов получения тонкие магнитные пленки могут иметь монокристаллическую либо поликристаллическую структуру. Известно, что поликристаллические структуры характеризуются гораздо большими значениями ширины линии ФМР, по сравнению с монокристаллическими. Главной причиной уширения линии ФМР в поликристаллических структурах обычно считается двухмагнонный механизм спинспиновой релаксации, вызванный структурными несовершенствами, такими как хаотичная взаимная ориентация зерен и морфология поверхности [7]. Внешние факторы определяются условиями осаждения ферромагнитного слоя и его последующей обработки. Вклад данных факторов вызывает особый интерес, поскольку режимы осаждения структуры и последующей обработки могут быть намеренно изменены [8]. В работах $[9,10]$ показано, что двухмагнонный механизм вносит наиболее значительный вклад в ширину линии ФМР, возрастающий при уменьшении толщины ферромагнитной пленки, в то время как для большинства структур вкладом внутреннего гильбертовского затухания можно пренебречь.

Другим важным вопросом является влияние одноосной магнитной анизотропии и обменного смещения на ширину линии ФМР для тонкопленочных структур вида ферромагнетик/антиферромагнетик (Ф/АФ). В работах $[10,11]$ получено, что при изменении толщины Ф-слоя ширина линии ФМР растет пропорционально квадрату величины обменного смещения вне зависимости от порядка осаждения $Ф$ - и АФ-слоев. В то же время при изменении толщины АФ-слоя не было обнаружено корреляции обменного смещения и ширины линии ФМР, несмотря на то, что и обменное смещение, и шири- 
на линии ФМР возрастают при увеличении толщины АФ-слоя [11,12].

В данной работе исследовано влияние внешних факторов на ширину линии ФМР в поликристаллических структурах вида Ф/АФ с обменным смещением при изменении толщины АФ-слоя и порядка осаждения $\Phi$ - и АФ-слоев. Обнаружено, что обменное смещение оказывает пренебрежимо малое влияние на ширину линии ФМР. В то же время вклад одноосной магнитной анизотропии, микроструктура и шероховатость границы раздела $\Phi$ - и АФ-слоев могут быть наиболее существенными внешними факторами.

\section{2. Изготовление и методы исследования образцов}

Методом магнетронного распыления были получены две серии многослойных структур вида $\mathrm{Ta} / \mathrm{NiFe} / \mathrm{IrMn} / \mathrm{Ta}$ (TS-структуры) и $\mathrm{Ta} / \mathrm{IrMn} / \mathrm{NiFe} / \mathrm{Ta}$ (BS-структуры). Структуры осаждались на подложку из монокристаллического кремния с кристаллографической ориентацией (100). Для улучшения адгезии и формирования микрокристаллической структуры последующих слоев на пластину кремния наносился буферный (затравочный) слой Та толщиной $30 \mathrm{~nm}$, далее проводилось осаждение $\Phi$ - и АФ-слоев в альтернативной последовательности. Для обеих серий образцов толщина ферромагнитного слоя $\mathrm{NiFe}$ составляла $10 \mathrm{~nm}$, а толщина антиферромагнитного слоя IrMn варьировалась в пределах 10-40 nm с шагом в $10 \mathrm{~nm}$. Для защиты от окисления и механических повреждений образцы покрывались сверху защитным слоем Та толщиной $30 \mathrm{~nm}$.

Перед осаждением образцов камера магнетрона откачивалась до давления $10^{-7}$ Torr. Осаждение образцов проводилось в аргоне при давлении $3.5 \cdot 10^{-3}$ Torr. Ферромагнитный слой $\mathrm{Ni}_{80} \mathrm{Fe}_{20}$ был получен путем соосаждения из мишеней $\mathrm{Ni}$ и $\mathrm{Fe}$, в то время как антиферромагнитный слой осаждался из мишени IrMn. Толщины слоев были определены из скоростей осаждения пленок. Скорости осаждения определялись по толщине калибровочных слоев, измеренной методом резерфордовского обратного рассеяния, и составляли порядка $3.7 \mathrm{~nm} / \mathrm{min}$.

Морфология поверхности образцов исследовалась методом атомно-силовой микроскопии (АСМ) на установке IntegraSpecta NT-MDT в полуконтактном режиме. Характеристикой образцов являлось полученное значение среднеквадратичной шероховатости $\sigma_{r m s}=\left\langle\Delta h^{2}\right\rangle^{1 / 2}$, где $\Delta h$ - отклонение высоты поверхности в данной точке сканирования от среднего значения, а также латеральная средняя площадь $\langle S\rangle$ топологических дефектов (островки и ямки на поверхности).

Магнитные свойства полученных образцов исследовались методом ферромагнитного резонанса с частотой $9.65 \mathrm{GHz}$. Постоянное магнитное поле ФМР (H на рис. 1), направленное вдоль поверхности образца, сканировалось в диапазоне до $6 \mathrm{kOe}$. Основные магнитные характеристики образцов получались из угловых зависимостей резонансного магнитного поля, $H_{r}(\alpha)$, где $\alpha-$ угол между направлением поля ФМР и направлением магнитного поля, приложенного при осаждении образца. Основные соотношения, связывающие магнитные характеристики и угловую зависимость $H_{r}(\alpha)$, приводятся ниже.

\section{3. ФМР в структурах с обменной Связью}

Запишем выражение для свободной энергии связанного с антиферромагнетиком ферромагнитного слоя

$$
\begin{aligned}
& F=2 \pi M_{S}^{2} \cos ^{2} \theta-M_{S} H \sin \theta \cos (\varphi-\alpha) \\
& -M_{S} H_{E B} \sin \theta \cos (\varphi-\beta)+\frac{M_{S} H_{K}}{2} \sin ^{2} \theta \sin ^{2}(\varphi-\eta),
\end{aligned}
$$

где $M_{S}$ - намагниченность в насыщении, $H_{K}, H_{E B}-$ поле одноосной и однонаправленной (обменное смещение) магнитной анизотропии. Обозначения углов поясняются на рис. 1. Ферромагнитный слой находится в плоскости $x y$, направление магнитного поля, приложенного при осаждении образца $H_{A}$ совпадает с осью $x$. Первый член в выражении (1) отвечает за энергию размагничивания - при ее расчете принимаем форм-фактор пленки равным: $N_{x}=0, N_{y}=0, N_{z}=4 \pi$.

Второй член в выражении для свободной энергии (1) отвечает за зеемановскую энергию взаимодействия намагниченности слоя с внешним магнитным полем, третий и четвертый члены - за энергию взаимодействия намагниченности с однонаправленной и одноосной анизотропией соответственно. Похожая форма для свободной энергии применялась, например, в статьях [13-15].

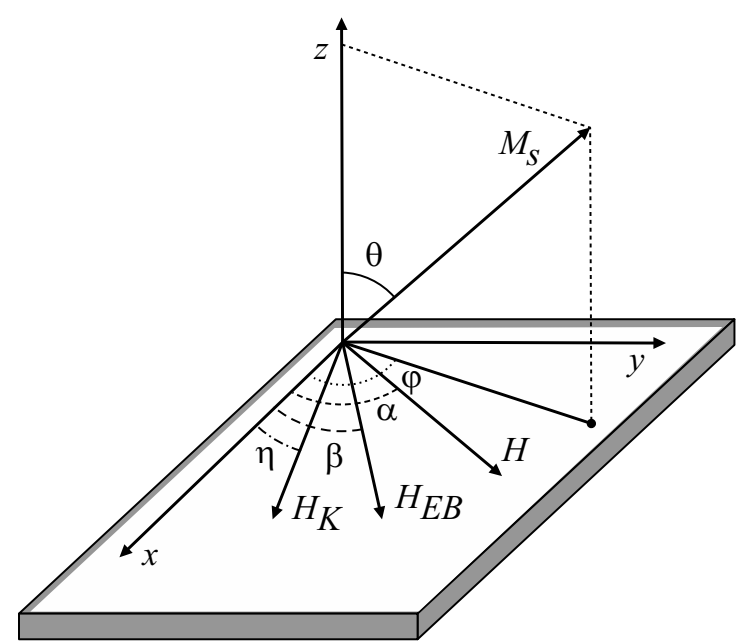

Рис. 1. Направления полей и намагниченности в ферромагнитном слое. 
Условие для резонанса

$$
\left(\frac{\omega}{\gamma}\right)^{2}=\frac{1}{M_{S}^{2} \sin ^{2} \theta}\left(F_{\theta \theta} F_{\varphi \varphi}-F_{\theta \varphi}^{2}\right)
$$

было получено независимо в целом ряде работ: [16-18].

Условия минимума для свободной энергии (1) $F_{\theta}=0$, $F_{\varphi}=0$ выполняются при значениях углов $\theta_{\min }=\frac{\pi}{2}$, $\varphi_{\min }=\alpha$ для соотношения полей $4 \pi M_{S} \gg H_{E B}+H_{K}$, которое выполняется в большинстве практических случаев. Подставляя вторые производные при значениях $\theta_{\min }, \varphi_{\min }$ в $(2)$, получаем

$$
\begin{aligned}
\left(\frac{\omega}{\gamma}\right)^{2}= & {\left[4 \pi M_{S}+H_{r}+H_{E B} \cos (\alpha-\beta)-H_{K} \sin ^{2}(\alpha-\eta)\right] } \\
& \times\left[H_{r}+H_{E B} \cos (\alpha-\beta)+H_{K} \cos 2(\alpha-\eta)\right]
\end{aligned}
$$

и резонансное поле $H_{r}$

$$
\begin{aligned}
H_{r}= & \frac{(\omega / \gamma)^{2}}{4 \pi M_{S}+H_{r}+H_{E B} \cos (\alpha-\beta)-H_{K} \sin ^{2}(\alpha-\eta)} \\
& -H_{E B} \cos (\alpha-\beta)-H_{K} \cos 2(\alpha-\eta) .
\end{aligned}
$$

Неколинеарность одноосной и однонаправленной анизотропий, $\beta \neq 0^{\circ}$, наблюдалась в ряде экспериментов [19]. Так в нашей работе [20] установлено, что угол неколинеарности между $H_{E B}$ и $H_{K}$ значителен, т. е. $\beta \neq 0^{\circ}$, при малых толщинах АФ-слоя менее $10 \mathrm{~nm}$ и постепенно уменьшается при бо́льших толщинах, в то время как $\eta$ близко к нулю при всех исследованных толщинах АФ.

Соотношения $(3,4)$ упрощаются, если за ось $x$ принять направление одноосной анизотропии, $\eta=0^{\circ}$ (или $180^{\circ}$, что не меняет соотношения $(3,4)$. Учитывая малость полей по сравнению с $4 \pi M_{S}\left(4 \pi M_{S} \gg H_{r}\right)$, получаем

$$
\left(\frac{\omega}{\gamma}\right)^{2}=4 \pi M_{S}\left(H r+H_{E B} \cos (\alpha-\beta)+H_{K} \cos 2 \alpha\right)
$$

и

$$
H r=\frac{(\omega / \gamma)^{2}}{4 \pi M_{S}}-H_{E B} \cos (\alpha-\beta)-H_{K} \cos 2 \alpha
$$

Аналогичные соотношения, но без учета угла неколинеарности $\beta$, были получены в работе [17].

Исходя из измеренных резонансных полей для ориентации внешнего поля ФМР вдоль и поперек оси легкой намагниченности, $H_{r}^{0}, H_{r}^{90}, H_{r}^{180}, H_{r}^{270}$, соответственно, для $\alpha=0^{\circ}, 90^{\circ}, 180^{\circ}$ и $270^{\circ}$ получаем следующее выражение для величины обменного смещения:

$$
H_{E B}=\frac{\left[\left(H_{r}^{270}-H_{r}^{90}\right)^{2}+\left(H_{r}^{180}-H_{r}^{0}\right)^{2}\right]^{1 / 2}}{2} .
$$

Данное выражение может использоваться для оценки величины обменного смещения с учетом неколинеарности одноосной и однонаправленной магнитной анизотропии. Однако в настоящей работе приводимые ниже величины полей были получены путем аппроксимации угловой зависимости резонансного поля $H_{r}(\alpha)$ выражением (6).

\section{4. Экспериментальные результаты}

Спектры ФМР позволяют определить значение резонансного поля $H_{r}$ и ширину резонансной линии ФМР $\Delta H$ (как разницу между максимумом и минимумом линии дифференциального спектра поглощения ФМР). Угловая зависимость резонансного поля $H_{r}(\alpha)$, может быть аппроксимирована соотношением (6) поскольку для экспериментальных данных выполняется условие $4 \pi M_{S} \gg H_{r}$ (так, значения $4 \pi M_{S}$ для исследованных образцов составляют порядка $11.5 \mathrm{kOe}$, в то время как значения $H_{r}$ составляют менее $\left.1 \mathrm{kOe}\right)$. Использование соотношения (6) позволяет определить значения резонансного поля в отсутствии анизотропии и обменного смещения, $H_{r 0}=(\omega / \gamma)^{2} /\left(4 \pi M_{S}\right)$, поля обменного смещения $H_{E B}$, поля одноосной анизотропии $H_{K}$ и угла неколинеарности $\beta$. Присутствие неколинеарности, $\beta \neq 0$, иллюстрируется на рис. 2 , где пунктирной линией изображена аппроксимирующая зависимость $H_{r}(\alpha)$ в отсутствие неколинеарности, а сплошной линией при $\beta=15^{\circ}$.

Ширина линии ФМР была измерена при значениях угла $\alpha$ взаимной ориентации внешнего поля ФМР и направления магнитного поля, приложенного при осаждении образцов, в 0 и 90 градусов. Эти два угла соответствуют измерениям при направлении магнитного поля вдоль ОЛН-образца и перпендикулярно ОЛНобразца. Результаты в зависимости от толщины АФ-слоя приведены на рис. 3. Обращают на себя внимание два обстоятельства. Первое - значительное расхождение в ширинах линий ФМР для TS- и BS-структур: $\Delta H_{T S}$ существенно меньше, чем $\Delta H_{B S}$. Второе - значения $\Delta H$ для $\alpha=0^{\circ}$ и $\alpha=90^{\circ}$ совпадают в пределах экспериментальной точности и синхронно повторяют вариации с толщиной. Исключение составляют результаты для BS-образцов с толщиной АФ-слоя $30 \mathrm{~nm}$.

Зависимость величины обменного смещения от толщины АФ-слоя для TS- и BS-структур показана на

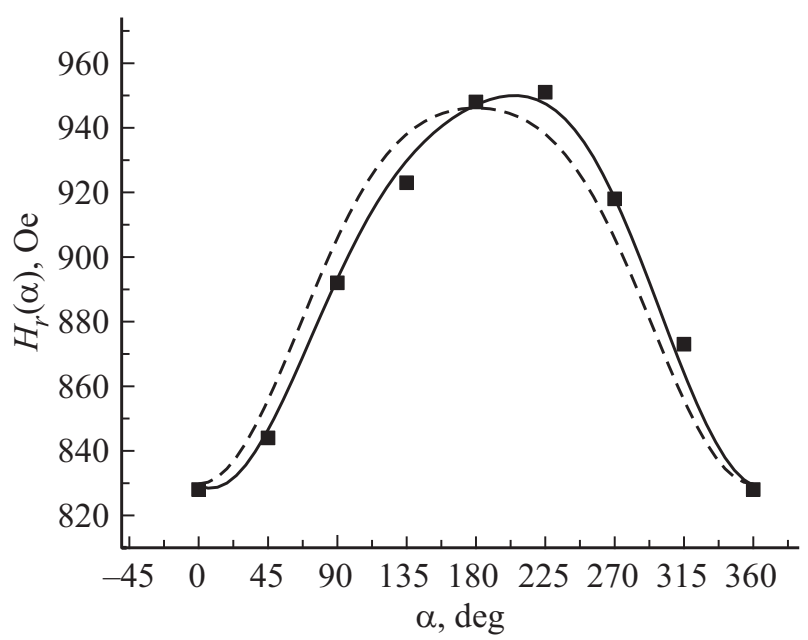

Рис. 2. Угловая зависимость поля ФМР для TS-образца с толщиной АФ-слоя $10 \mathrm{~nm}$. Линиями показана аппроксимация экспериментальных результатов соотношением (6) (с $\beta=0-$ пунктирная линия и $\beta=15^{\circ}-$ сплошная линия). 
рис. 4, $a$. Несмотря на некоторые количественные различия, качественный вид зависимостей совпадает для обоих типов структур.

Зависимость шероховатости поверхности образцов обоих типов от толщины АФ-слоя показана на рис. $4, b$. Из рисунка видно, что значение $\sigma_{r m s}$ для TS-образцов больше, чем для BS. Также можно заметить, что зависимости значения $\sigma_{r m s}$ от толщины АФ-слоя в TS- и BS-структурах находятся в противофазе.

Более детальное исследование влияния среднеквадратичной шероховатости поверхности образцов на величину обменного смещения приведено в работе [21].

\section{5. Обсуждение результатов}

Важно отметить, что свойства двухслойных структур вида $\Phi / А \Phi$ с различным порядком осаждения $\Phi$ - и АФ-слоев могут различаться из-за различий механизмов роста слоев [11,22]. В TS-структурах Ф-слой осаждается непосредственно на буферный слой Та, в значительной степени повторяя его шероховатость. При увеличении толщины АФ-слоя в структурах такого типа микроструктурные параметры Ф-слоя, такие как размер зерна
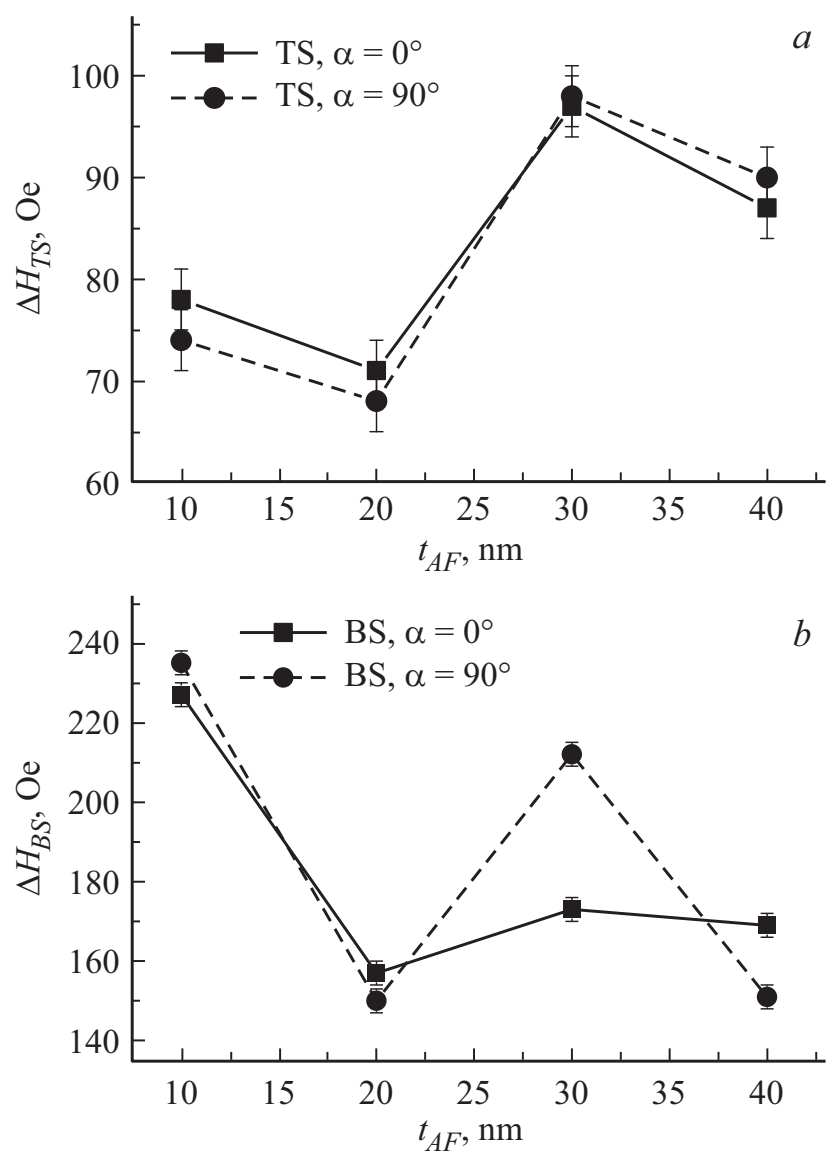

Рис. 3. Зависимость ширины линии ФМР для параллельной и перпендикулярной ориентации ОЛН образца относительно внешнего поля ФМР от толщины АФ-слоя в ТS $(a)$ и $\mathrm{BS}(b)$ образцах.
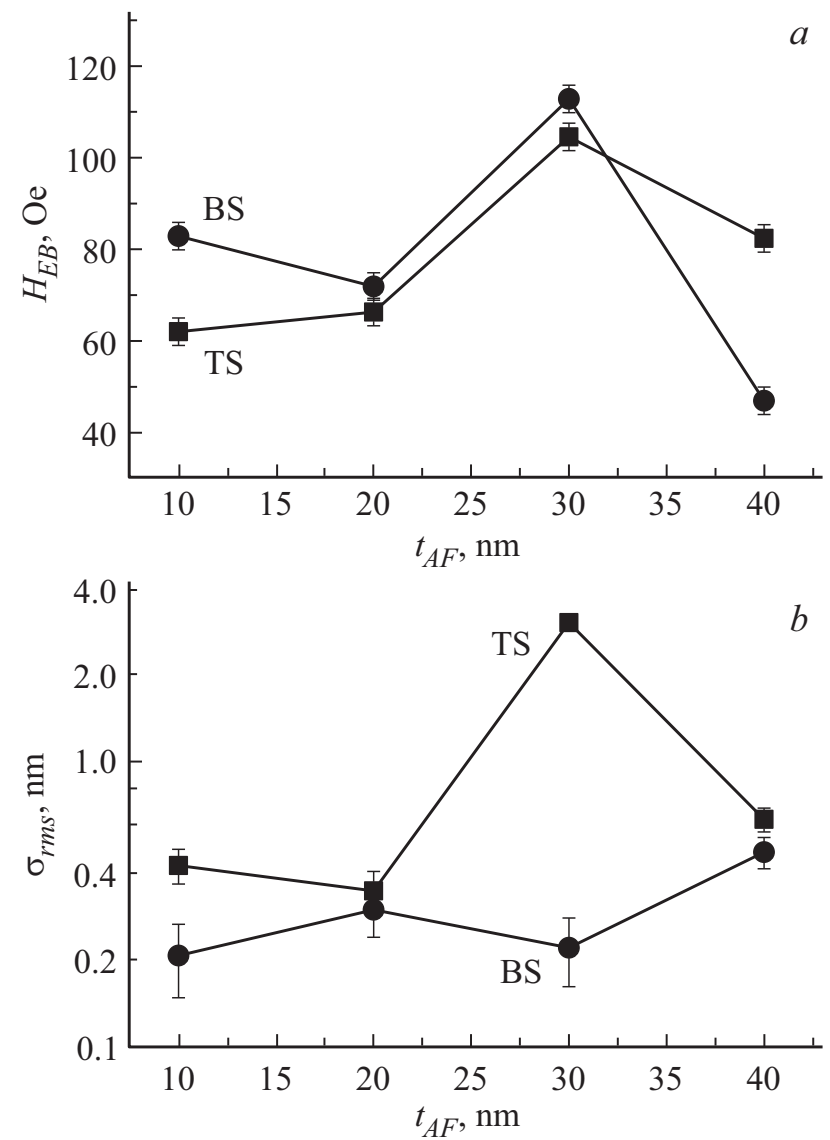

Рис. 4. Зависимость обменного смещения в TS- и BS-структурах от толщины АФ-слоя $(a)$. Зависимость среднеквадратичной шероховатости поверхности TS- и BS-структур от толщины АФ-слоя $(b)$.

и текстура, не претерпевают существенных изменений. В то же время величина обменного смещения может изменяться из-за изменения анизотропии АФ-слоя при изменении его толщины [23].

В случае, если поле обменного смещения направлено вдоль легкой оси образца $\left(\beta=0^{\circ}\right)$, вклад однонаправленной анизотропии $H_{E B}$ в величину резонансного поля $H_{r}$ может быть существенным при измерениях вдоль легкой оси и должен отсутствовать при измерениях перпендикулярно легкой оси, в соответствии с уравнением (6). Аналогичного эффекта можно ожидать и для ширины линии ФМР. Тот факт, что ширины линий ФМР для $\alpha=0^{\circ}$ и для $\alpha=90^{\circ}$ в случае TS-структур практически совпадают (рис. $3, a$ ), говорит о том, что, либо обменное смещение отсутствует, либо влияние обменного смещения на ширину линии ФМР пренебрежимо мало по сравнению с влиянием других факторов. Поскольку первое предположение опровергается рис. 4, $a$, следует признать, что справедливо последнее. Однако, это противоречит выводам, содержащимся в некоторых других публикациях.

Так, линейная зависимость ширины линии ФМР $\Delta H$ от $H_{E B}$ была предложена в работе [12], где предпо- 
лагалось, что эффективное поле в Ф-слое изменяется пропорционально обменному полю, создаваемому в $\Phi$-слое поли-, микро- или нанокристаллическим слоем АФ. Квадратичная зависимость $\Delta H \sim H_{E B}^{2}$ была выведена в работах $[4,10]$, основанных на подходе Ариаса и Миллса [24]. В этом подходе уширение линии ФМР является результатом флуктуации обменной связи из-за шероховатости границы раздела Ф/АФ. Наши результаты показывают, что, несмотря на большие вариации шероховатости (рис. $4, b$ ) и обменного смещения (рис. $4, a$ ) в образцах TS, ширины линии ФМР для $\alpha=0^{\circ}$ и $\alpha=90^{\circ}$ в пределах статистических ошибок совпадают (рис. 3, $a$ ) и, следовательно, значительного влияния $H_{E B}$ на ширину линии ФМР не наблюдается.

В то же время влияние шероховатости поверхности образцов на ширину линии ФМР достаточно заметно, о чем свидетельствует рис. 5. Сплошная линия на рисунке представляет собой аппроксимацию экспериментальных данных по ширине линии ФМР, $\Delta H$, для серии TS-образцов зависимостью, которую можно записать в виде

$$
\Delta H_{T S} \propto \sqrt[3]{\sigma_{r m s} S}
$$

Здесь: $S$ - средняя площадь дефектов, значение $\sqrt[3]{\sigma_{r m s} S}$ представляет собой средний линейный размер дефектов на поверхности образца. На рис. 5 показано, что экспериментальные данные хорошо описываются такой зависимостью между $\Delta H$ и средним размером морфологических дефектов поверхности. Это согласуется с известными результатами [25], где продемонстрирована связь ширины линии ФМР с размером зерна, и [26], где предсказана линейная связь между $\Delta H$ и радиусом пор на поверхности образца. В других работах предсказана линейная зависимость $\Delta H$ от удельного объема пор [7] и площади дефектов $[27,4,10,28]$ на поверхности образца. При этом экспериментальные данные о зависимости ширины линии ФМР с топологическими дефектами на поверхности или интерфейсе многослойных структур практически отсутствуют.

В образцах $\mathrm{BS}$, где Ф-слой растет на слое АФ, микроструктура АФ-слоя может влиять на свойства осажденного на него Ф-слоя. Увеличение толщины слоя АФ может привести, например, к увеличению размера зерен АФ в объеме и на границе раздела АФ/Ф. Нанесенный сверху слой $\Phi$ принимает типичный размер зерна АФ, т.е. размер зерна $Ф$, возможно, увеличивается с увеличением толщины слоя АФ. Значительно большие абсолютные значения ширины линии ФМР для образцов BS (по сравнению с TS) подтверждают это предположение (рис. 3, $a$ и 3,b). Предполагается, что уширение линий ФМР для BS-структур по сравнению с TS-структурами не связано с обменным смещением. Подтверждение незначительности влияния обменного смещения следует из рис. $3, b$, где $\Delta H_{B S}$ для $\alpha=0^{\circ}$ либо близко, либо меньше, чем для $\alpha=90^{\circ}$, за исключением образца с $t_{A F}=40 \mathrm{~nm}$. Кроме того, было показано, что шероховатость поверхности BS-образцов меньше, чем $\mathrm{TS}$ (рис. 4, $b$ ), что говорит о том, что шероховатость также не является причиной уширения линии ФМР.

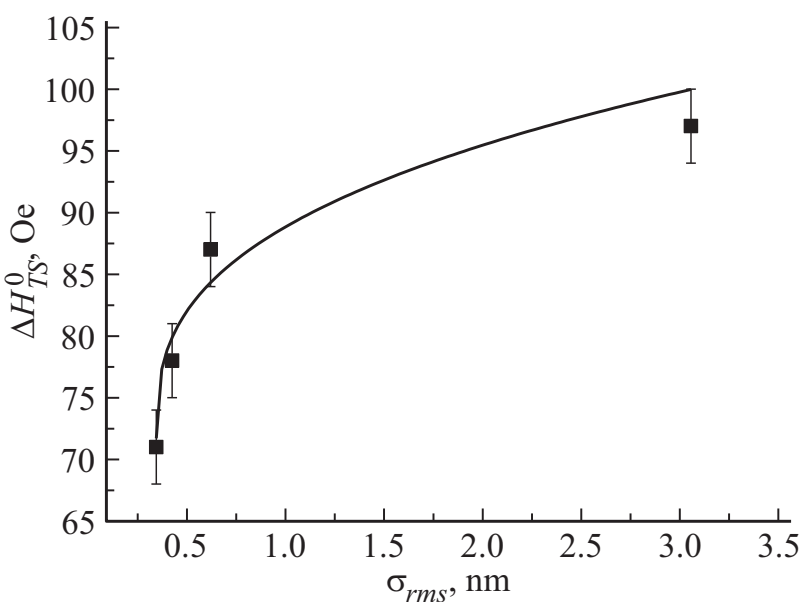

Рис. 5. Зависимость ширины линии ФМР от среднеквадратичной шероховатости поверхности TS-образцов. Сплошная линия является аппроксимацией экспериментальных данных соотношением (8)

Этот краткий анализ дает основание утверждать, что единственным фактором, влияющим на уширение $\Delta H$ при наложении поля вдоль трудной оси по сравнению с легкой осью в образцах $\mathrm{BS}$, является одноосная магнитокристаллическая анизотропия $H_{K}$. Учитывая, что образцы имеют поликристаллическую структуру, мы предполагаем, что работает приближение сильно связанных зерен с $H_{K} \ll 4 \pi M_{s}$. В этом приближении поликристалл рассматривается как однородная среда с неоднородным эффективным полем, на которое влияет наличие зерен с различной кристаллографической ориентацией. Магнитные флуктуации зерен связаны друг с другом через размагничивающие поля [7], что приводит к нескомпенсированной кристаллографической анизотропии, способствующей усилению одноосной анизотропии и вызывающей плавные пространственные колебания намагниченности. Пространственно-флуктуирующая намагниченность, так называемая „микромагнитная рябь (ripple)“, наблюдающаяся в лоренцевской просвечивающей микроскопии $[29,30]$, коррелирует с размером зерна [23]и может быть если не основным, то дополнительным источником уширения линии ФМР [31]. Основываясь на модели Ариаса и Миллса [19], и используя подход Резенде и др. [10], примененный для оценки уширения линии ФМР под влиянием обменного смещения, экспериментальная зависимость ширины линии ФМР, $\Delta H$, может быть аппроксимирована квадратичной зависимостью от одноосной анизотропии $H_{K}$. Квадратичная зависимость $\Delta H\left(H_{K}\right)$ была получена в работе [20]. Для выделения на фоне других конкурирующих вкладов, на рис. 6 квадратичная зависимость представлена в виде разности ширин ФМР линий для $\alpha=90^{\circ}$ и $\alpha=0^{\circ}$ как

$$
\Delta H_{B S}^{90}-\Delta H_{B S}^{0}=A H_{K}^{2}+B,
$$

где $A$ и $B$ подгоночные параметры. Как видно из рис. 6, данное соотношение хорошо описывает эксперименталь- 


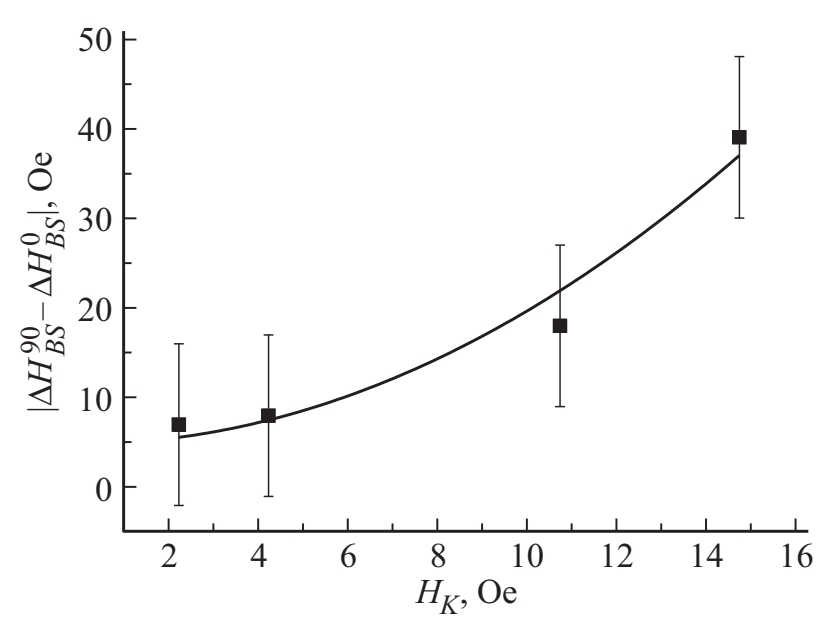

Рис. 6. Зависимость разности ширин линий ФМР для BS-образцов. Сплошной линией показана аппроксимация экспериментальных результатов соотношением (9).

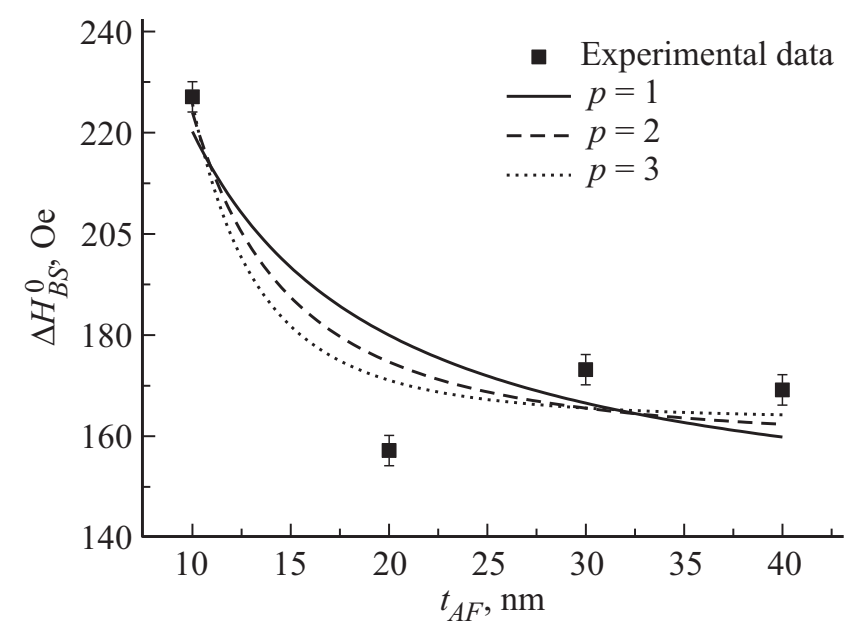

Рис. 7. Зависимость ширины линии ФМР от толщины АФ-слоя для BS образцов. Линии приведены в соответствии с уравнением (10).

ные данные для BS-структур при значениях параметров $A=0.15 \pm 0.02, B=4.8 \pm 2.4$.

Таким образом, показано, что единственным фактором, влияющим на уширение $\Delta H$ для трудной оси по сравнению с легкой осью в образцах $\mathrm{BS}$, является наличие пространственных осцилляций одноосной магнитокристаллической анизотропии, которые, как мы предполагаем, связаны не с шероховатостью, как предложено в работе [10], а с не полностью скомпенсированной магнитокристаллической анизотропией поликристаллического Ф-слоя в BS-структуре, как предложено в работе [23]. В отличие от BS-, в TS-структуре подобный анализ выполнить невозможно ввиду меньших абсолютных значений ширин и их разности $\Delta H_{T S}^{90}-\Delta H_{T S}^{0}$, рис. $3, a$.

Исследования зависимости ширины линии ФМР от толщины слоя антиферромагнетика в структуре типа АФ/Ф весьма немногочисленны [12]. Гораздо больше результатов было опубликовано по зависимости ФМР от толщины слоя ферромагнетика, $\propto 1 / t_{F}^{p}$, с разными показателями $p: p=1$ в работах $[10,12,32]$, что соответствует затуханию намагниченности за счет рассеяния на поверхностных (межфазных) дефектах, $p=1.5 \pm 0.2$ в [19] и $p=3$, предложенными для более толстых ферромагнитных пленок в рамках двухмагнонного механизма рассеяния [33].

На рис. 7 приведены экспериментальные данные по зависимости $\Delta H_{B S}^{0}$ от толщины слоя антиферромагнетика и проведены кривые, соответствующие зависимости $1 / t_{A F}^{p}$ : для разных значений $p$ :

$$
\Delta H_{B S}^{0}\left(t_{A F}\right)-\Delta H_{B S}^{0}\left(t_{A F}=\infty\right) \propto \frac{1}{t_{A F}^{p}} .
$$

При значениях показателя $p=1,2$ и 3 ширины линий ФМР для массивного АФ-слоя составляют, соответственно, $\Delta H_{B S}^{0}\left(t_{A F}=\infty\right)=140 \pm 19, \quad 158 \pm 10$, $163 \pm 7$ Oe.

Значения показателей степени для $\Phi$ и АФ находятся в одном диапазоне. Это указывает на общую причину такой зависимости как при изменении толщины Ф-слоя, так и при изменении толщины АФ-слоя, так как в обоих случаях происходит изменение микроструктуры (рост размера зерна) с увеличением толщины слоя. Рост размера зерна провоцирует увеличение угловой дисперсии однонаправленной анизотропии АФ-слоя и угловой дисперсии намагниченности АФ-слоя, что приводит к росту ширины линии ФМР, как показано в работе [23].

\section{6. Заключение}

Внешние факторы, влияющие на ширину линии ФМР, были исследованы в системах Ф/АФ с различным порядком осаждения слоев (TS, BS). Вклада однонаправленной анизотропии в ширину линии ФМР в исследуемых образцах не наблюдалось. Ширина линии ФМР в ТSобразцах увеличивалась пропорционально среднему размеру дефектов на поверхности образцов. Для структур BS такой зависимости не наблюдалось, что можно объяснить гораздо меньшими значениями среднеквадратичной шероховатости поверхности образца и, по-видимому, более гладкой поверхностью слоя АФ на границе раздела АФ- и Ф-слоев.

В структурах вида АФ/Ф было обнаружено, что в ширину линии ФМР, измеренной вдоль трудной оси, значительный вклад вносит не однонаправленная, а одноосная анизотропия. Разница в значениях ширины линий ФМР, измеренных вдоль трудной и легкой оси, изменяется пропорционально квадрату $H_{K}$.

Ширина линии ФМР вдоль легкой оси в образцах BS уменьшается с увеличением толщины слоя АФ примерно, как $1 / t_{A F}^{p}$, при $p=1-3$. Сходная форма зависимости ширины линии ФМР от толщины Ф- и АФ-слоев свидетельствует о сходстве внешних факторов, формирующихся в слое $\Phi$ при росте слоев. Мы предполагаем, что это микроструктурные факторы, такие как размер зерна. 


\section{Финансирование работы}

Работа выполнялась в рамках государственного задания тема № 01201268472.

\section{Конфликт интересов}

Авторы заявляют, что у них нет конфликта интересов.

\section{Список литературы}

[1] E. Schlomann, R. Tutison, J. Weissman, H.J. Van Hook, T. Vatimos. J. Appl. Phys. 63, 3140 (1988).

[2] R.E. Camley, D.L. Mills. J. Appl. Phys. 82, 3058 (1997).

[3] N. Cramer, D. Lucic, R.E. Camley, Z. Celinski. J. Appl. Phys. 87, 6911 (2000).

[4] D.L. Mills, S.M. Rezende. Spin Dynamics in Confined Magnetic Structures II. Topics Appl. Phys. 87, 27-59 (2003) / Ed. B. Hillebrands, K. Ounadjela. Springer-Verlag, Berlin, Heidelberg (2003).

[5] M. Sparks M, R. Loudon, C. Kittel. Phys. Rev. 122, 791 (1961).

[6] R.C. LeCraw, E.G. Spencer, C.S. Porter. Phys. Rev. 110, 1311 (1958).

[7] A.G. Gurevich, G.A. Melkov. Magnetization Oscillation and Waves. CRC Press, N. Y. (1996).

[8] B. Heinrich: Ferromagnetic resonance in ultrathin structures / Eds B. Heinrich, J.A.C. Bland. Ultrathin magnetic structures II. Springer, Berlin, Heidelberg (1994). Ch. 3.

[9] B.K. Kuanr, R.E. Camley, Z. Celinski. J. Appl. Phys. 93. 7723 (2003).

[10] S.M. Rezende, A. Azevedo, M.A. Lucena, F.M. de Aguiar. Phys. Rev. B 63, 214418 (2001).

[11] S. Yuan, B. Kang, L. Yu, S. Cao, X. Zhao. J. Appl. Phys. 105, 063902 (2009).

[12] W. Stoecklein, S.S.P. Parkin, J.C. Scott. Phys. Rev. B 38, 6847 (1988).

[13] A. Layadi, W.C. Cain, J.-W. Lee, J.O. Artman. IEEE Transact. Magn. 23, 2993 (1987).

[14] M. Díaz de Sihues, C.A. Durante-Rincón, J.R. Fermin. JMMM 316, e462 (2007).

[15] R.D. McMichael, M.D. Stiles, P.J. Chen, W.F. Egelhoff. Jr. Phys. Rev. B 58, 8605 (1998).

[16] H. Suhl. Phys. Rev. 97, 2, 555 (1955).

[17] J. Smit H., H.G. Beljers. Phillips. Res. Rep. 10, 1, 113 (1955).

[18] T. Gilbert, A Lagrangian. Phys. Rev. 100, 1243 (1955).

[19] H.C. Choi, C.Y. You, K.Y. Kim, J.S. Lee, J.H. Shim, D.H. Kim. Phys. Rev. B 81 (2010) 224410.

[20] I.O. Dzhun, G.V. Babaytsev, N.G. Chechenin, Ch.A. Gritsenko, V.V. Rodionova. JMMM 470, 151 (2019).

[21] Е.И. Шанова, И.О. Джунь, Н.Г. Чеченин. Перспективные материалы (Неорган.материалы: Прикладные исследования). 11, 5 (2013). E.I. Shanova, I.O. Dzhun, N.G. Chechenin. Perspect. Mater. Inorganic Mater: Appl. Res. 5, 2, 89 (2014).

[22] Е.В. Хоменко, Н.Г. Чеченин, И.О. Джунь, Н.С. Перов, В.В. Самсонова, А.Ю. Гойхман, А.В. Зенкевич. ФТТ 52, 1583 (2010).

[23] K. O'Grady, L.E. Fernandez-Outon, G. Vallejo-Fernandez. J. Magn. Magn. Mater. 322, 883 (2010).

[24] R. Arias, D.L. Mills. Phys. Rev. B 60, 7395 (1999).
[25] R.C. LeCraw, E.G. Spencer, C.S. Porter. Phys. Rev. 110, 6, 1311 (1958).

[26] M. Sparks, R. Loudon, C. Kittel. Phys. Rev. 122, 3, 791 (1961).

[27] R. Arias, D.L. Mills. Phys. Rev. B 60, 10, 7395 (1999).

[28] B.K. Kuanr, R.E. Camley, Z. Celinski. J. Appl. Phys. 95, 6610 (2004).

[29] N.G. Chechenin, C.B. Craus, A. Chezan, T. Vystavel, D.O. Boerma, J.Th.M. De Hosson, L. Niesen. IEEE Trans. Magn. MAG 38, 5, 3027 (2002).

[30] N.G. Chechenin. J. Magn. Magn. Mater. 300, 198 (2006).

[31] Н.Г. Чеченин. ФТТ 46, 3, 466 (2004).

[32] V. Speriosu, S. Parkin, C. Wilts. IEEE Trans. Magn. MAG 23, 5, 2999 (1987).

[33] R.D. McMichael, M.D. Stiles, P.J. Chen, W.F. Egelhoff Jr. J. Appl. Phys. 83, 7037 (1998).

Редактор Т.Н. Василевская 